\title{
Pacing Strategies and Emotional Pattern During Intermittent 3-min runs in Secondary School Participants
}

\author{
Bertrand Guilloux and Bertrand Baron* \\ IRISSE Engineering Research and Intervention, Sport, Health and Environment (EA 4075), Université de La Réunion, France
}

Received: February 02, 2015; Accepted: April 29, 2015; Published: June 01, 2015

*Corresponding author: Bertrand Baron, STAPS Department, Université de La Réunion, 117 rue du Général Ailleret, 97430 Le Tampon, France, Tel: +33-262-579-573; Fax : +33-262-579-571; Email: bertrand.baron@univ-reunion.fr

\begin{abstract}
The aim of this study was to analyse pacing strategy and emotional regulation during intermittent 3-min runs. 127 school participants performed 2 exhaustive intermittent 3-min runs at different days. The effort, the pleasure, the desire to continue, the desire to stop and the level of resources were measured every $100 \mathrm{~m}$ with Likert scales from 0 to 10. The CONTROL group was asked to realize 2 runs as fast as possible without any other consign. The RPE group was asked to realise the first run with the same consign but has to start the second run at a minimal RPE level corresponding to the average RPE of the first run. For this, subjects were asked to self-select the intensity which induced the expected RPE. Then, exercise is totally self-paced.

The strategy, which consists in a slow start with an increase of speed at the end, could be observed, for each test of each group. Fluctuations of running intensities exist, except during the second run of the RPE group. An important emotional reserve was observed in each condition. But this reserve was lower during the second run for the RPE group, contributing to the increase in performance during the second run for the RPE group $(+13.5 \%)$, whereas it was not significantly different for the CONTROL group.
\end{abstract}

For each group and each test, effort, affective balance and desire to stop significantly increased between the first and the last repetition, whereas pleasure, desire to continue and available resources significantly decreased during running. The most important observation is that the emotional pattern corresponded to a linear evolution in each condition whereas exercise is self-paced. This highlights that emotional regulation is a part of pacing strategy.

Finally, we define the motivation to pursue a tired exercise as the level of acceptance of effort. We propose that the ratio between the desire to continue and the level of effort could be considered as a good marker of the motivation in this context.

Keywords: Pacing strategy; Emotions; Motivation; Perceived exertion

\section{Introduction}

The ability to accurately self-pace an exercise bout is a feature of race and time trial performance $[1,2]$. Research on pacing strategy during exercise is the 'unexplored territory' in sports performance [3]. This statement is especially true in physical education.
Elite athletes optimally monitor the intensity of exercise in order that their energy-producing capacities are not exhausted before they reach the finish line [4]. Hettinga et al. [5] have suggest that athletes develop a stable template of the power outputs they can sustain during exercise of different durations or distances. The optimum pacing strategy requires the presence of an accurate pre-established estimate of the power output that can be sustained for the duration of the event [5]. This process has been described as an internal negotiation, which requires an estimation of the magnitude of the task remaining, the momentary aerobic power output and the remaining anaerobic energetic reserves amongst other inputs [6]. Pacing strategies can be thought of as a complex process in which the subject controls the intensity of exercise at any time by taking into consideration physiological and psychological reserves in relation to the estimated time until the finish [7] at that exercise intensity.

During exercise performed at severe intensity, what prevents the occurrence of catastrophic fatigue is the interaction of a protective mechanism that precedes the failure in physiological systems and manifests itself as powerful perception of fatigue and displeasure $[8,9]$.

Emotional process is of great importance in pacing strategy [8]. Individuals develop strategies that allow them to reach their goals, and mobilize and monitor their behaviours in order to attain their goals [10]. Emotional phenomena represents central mechanisms of self-regulation that help humans deal effectively with their environments [10-12].

The study of Lander et al. [13] demonstrates that self-paced exercise pose a reduced metabolic challenge when compared with matched-intensity enforced constant paced submaximal exercise. It is likely that this is attributable to the ability to voluntary fluctuate power output in accordance with transient sensations of fatigue during the exercise bout. The voluntary behavioural change fluctuate pace is therefore a conscious decision based on subconscious physiological feedback from array of peripheral receptors. This suggests that pacing is a mechanism to minimize the conscious sensations of fatigue experienced during exercise, which enables homeostasis to be defended during exercise. 
Ekkekakis et al. [9] consider that pleasure to signify utility and displeasure to signify danger. Because low-intensities are associated with pleasure, it tends to evoke approach reactions. On the opposite high intensities tend to evoke withdrawal reactions because it is associated with displeasure [9]. Indeed, when an athlete selects high exercise intensity, he must also accept that the sensation of effort will have to increase progressively until the finish line. This requires that the motivation level is sufficient in order to maintain the expected power output [8].

Sports teachers and coaches need for easy tools to measure the motivation of subjects. Unfortunately, measure motivation to continue an exercise despite the occurrence of psychophysiological mechanisms of fatigue is not easy and no psychometric scale was designed for it.

Nevertheless, Gendolla and Richter [14] have proposed that effort refers to the intensity aspect of motivation. But the motivational processes that allow the athlete to control its intensity in relation to the perceived sensations are complex. It is difficult to imagine that the study of a single parameter such as perceived exertion can allow a complete analysis of motivational processes. Thus, we have recently used other emotional parameters in addition to the perceived exertion to better understand the process of pacing strategy during intermittent sprints [15]. These parameters were pleasure, the desire to continue the exercise, the desire to stop the exercise and the available level of resources.

The first aim of the present study was to test whether the use of these parameters may allow an estimation of motivation to continue a tiring exercise.

The second aim of this study was to test the effect of a start to a higher perceived exertion than spontaneously chosen on pacing strategy, emotional regulation and motivation.

It is well known that well-trained athlete have the ability in this complex skill [16] whereas inexperienced subjects are unable to pace themselves effectively $[17,18]$. Indeed, the process of pacing strategy amounts to make compatible the nature of the task and the personal psycho-physiological capacities [8]. This process is based on representations. When a subject has no prior experience of a particular exercise, the representations of the task are imprecise because no element of comparison from a prior event is available in the memory. In this context, the expected time to cover a distance cannot be precisely estimated. Likewise, physiological capabilities and the capacity to resist the sensations of fatigue are not yet known. As a result, the optimum pacing strategy cannot be determined either before or during the exercise bout. Herbert et al. $[19,20]$ have shown that there is a strong association between interoceptive sensitivity and the intensity of emotional experience, which corresponds to the quantity of memorised emotions. In accordance, it is argued that cognitive information developed by each training session would reinforce the interoceptive sensitivity and the physiological awareness [8]. For this reason, self-paced exercises are often used in Physical Education in order to improve the capacity to control exercise intensity in relation with personal psychophysiological capacities.
In a recent study, Foster et al have shown that the pattern of learning the performance template is primarily related to increased confidence that the trial can be completed without unreasonable levels of exertion or injury [21]. Pacing strategies can be thought of as a complex process in which the subject controls the intensity of exercise at any time by taking into consideration physiological and psychological reserves in relation to the estimated time until the finish [7] at that exercise intensity [8].

The assumption of Gendolla and Richter [14] is that all our behaviour is guided by the energy conservation principle. Indeed, athlete has to confront the fact that enough energy is mobilized to cope with obstacles in goal attainment process and the fact that enough energy has to be conserved to reduce the probability of depletion and exhaustion.

Accordingly, we hypothesised that this principle would impose that an emotional reserve exists at the end of exercise in school participants when the intensity is self-paced even when maximal performance is asked because the maximal personal capacities are unknown. Indeed, if psycho-physiological process of fatigue could be envisaged as protective mechanisms, it could be hypothesised that pacing strategy would correspond to a protective behaviour in novice. Only experience could induce that this emotional reserve will be reduced, when subject becomes aware that more important effort could be supported for the duration of the task.

We hypothesise that a more important effort than spontaneously mobilized may be supported by the school participants. Moreover, we expect that the method, which consists in imposing a more important effort at the beginning, would improve performance.

Finally, according to St Clair Gibson et al. [16], pacing strategy is acquired during adolescence and is a stable process. The pacing strategy is dependent on the pre-established template and considerable time and effort may be required to change a specific strategy that has been followed frequently [22]. St Clair Gibson et al. [23] have suggested that the motor sequences representative of the performance template are almost certainly programmed into the motor cortex from prior athletic performance, potentially from childhood Thus, an interesting possibility is that training methods could be designed, even during childhood, to improve the efficiency and stability of the pacing strategies.

Recently, we showed that emotional responses were controlled during intermittent sprints in order that effort could be maintained until the end whether during level, downhill and uphill running in young adults [15]. Results have shown that the gradient of the running surface did not influence the pattern of emotional responses, which corresponds to a quasi-maximal level of emotional reserve at the beginning and a low level at the end of exercise.

In accordance, we suggest that a pre-established template in emotional responses would exist in school participants. We hypothesize that it would remain constant even when a faster start is imposed at the beginning of the exercise. 


\section{Methods}

\section{Experimental approach to the problem}

Pacing strategies were analysed during 2 exhaustive 3-min runs for a control group and a test group. Effort was measured using Borg's category ratio scale [24], which consists of 11 statements ranging from 0 to 10 (from "nothing" to "maximum"). This scale was used in order to quantify all the other emotional parameters as previously proposed [15]: that is, the pleasure, the desire to continue, the desire to stop, and the available resources. The affective balance (AB) was calculated as the difference between effort and pleasure [15].

\section{Participants}

127 secondary school participants have realized running tests during their sport classes. Participants aged $15.2 \pm 1.0$ year. They were taught to use the Rating of Perceived Exertion (RPE) scale [24] to monitor their exercise intensities during precedent classes. Thus, all participants were accustomed to quantifying their individual RPE, but also other emotional parameters [15] for 1 training session when running. Local ethics committee has approved this work.

\section{Procedures}

A CONTROL group (56 participants) and a RPE group (71 participants) performed an incremental test (test 1) and 2 exhaustive 3-min runs (tests 2 and 3). During these 3-min runs, participants were asked to realize the most important performance as possible. They were stopped at each $100 \mathrm{~m}$ for $1 \mathrm{~min}$ in order to respond to the emotional questioner. During these arrests, the chronometer was stopped.

The CONTROL group was asked to realize their 2 runs as fast as possible without any other consign. The RPE group was asked to realise the first run with the same consign but has to start the second run at a minimal RPE level corresponding to the average RPE of the first run.

For this, subjects were asked to self-select the intensity which induced the expected RPE. Then, exercise is totally self-paced.

The emotional parameters were collected every $100 \mathrm{~m}$. Running tests were performed on a $100 \mathrm{~m}$ track. The speed was measured every $100 \mathrm{~m}$ for the firsts $500 \mathrm{~m}$. Then, the speed was measured for the last $100 \mathrm{~m}$.

\section{Statistical analysis}

Calculations were performed using XLSTAT. Standard statistical methods were used for the calculation of mean and standard deviations. Student test was used to determine significant differences of measured variables between each running condition.

ANOVA with repeated measurements was used to compare the changes in parameters during the tests. Normal Gaussian distribution of the data was verified by the Shapiro-Wilks test. Compound symmetry, or sphericity, was verified by the Mauchley test. Multiple comparisons were made with the Tukey HSD post hoc test when the Greenhouse-Geisser epsilon correction factor was $>0.50$, or with the Bonferroni post hoc test when the epsilon was $<0.50$.

The Pearson's coefficient was computed to test linear correlation among variables measured at the end of each repetition.

Statistical significance was set at a level of $p=0.05$ for all analyses.

\section{Results}

\section{Test 1: Incremental test}

VAM was $12.40 \pm 1.67 \mathrm{~km} \cdot \mathrm{h}^{-1}$ for the group CONTROL and $12.14 \pm 1.81 \mathrm{~km} \cdot \mathrm{h}^{-1}$ for the group RPE.

During the incremental test, effort, pleasure, desire to continue, desire to stop and the level of resources are correlated to intensity $\left(\mathrm{R}^{2}=0,65 ;-0,18 ;-0,24 ; 0,41\right.$ et $-0,47$, respectively, $P$ $<0.05$ ).

\section{Test 2 and 3: first and second 3-min run for the group CONTROL and the group RPE}

Average distance, speed and emotional parameters of the two groups for the two races were presented in Table 1.

\section{Evolution of self-selected speed}

Test 1 group RPE: Self selected speed significantly increased between the start and the finish of the run $(p<0.05)$ but significantly decreased between the start and the $500 \mathrm{~m}(p$ $<0.05$ ). Post-hoc tests show significant differences between all measures (Figure 1).

Test 2 group RPE: Self-selected speed significantly increased between the start and the stop of the run $(p<0.05)$ but did not significantly evolve between the start and the $500 \mathrm{~m}(p<0.05)$. Post-hoc tests show significant differences between the $500 \mathrm{~m}$ and the finish (Figure 2).

Test 1 group CONTROL: Self-selected speed significantly increased between the start and the stop of the run $(p<0.05)$ and between the start and the $500 \mathrm{~m}(p<0.05)$. Post-hoc tests show significant differences between all the measures (Figure 3).

Test 2 group CONTROL: Self-selected speed significantly increased between the start and the stop of the run $(p<0.05)$ and between the start and the $500 \mathrm{~m}(p<0.05)$. Post-hoc tests show significant differences between all the measures (Figure 4).

\section{Relations between time and emotional parameters}

Test 1 group RPE: Effort, affective balance and desire to stop significantly increased between the first and the last repetition $(1.73 \pm 1.37$ vs $7.41 \pm 2.15, p<0.05 ;-4.62 \pm 2.70$ vs $4.27 \pm 2.27, p$ $<0.05 ; 1.85 \pm 2.50$ vs $6.02 \pm 3.24, p<0.05$, respectively), whereas pleasure, desire to continue and available resources significantly decreased during running $(6.35 \pm 3.69$ vs $3.14 \pm 2.63, p<0.05$; $6.82 \pm 3.25$ vs $2.89 \pm 2.51, p<0.05 ; 7.80 \pm 2.93$ vs $3.17 \pm 2.53, p<$ 0.05 , respectively). 
Table 1: Average distance, absolute speed $\left(\mathrm{km} . \mathrm{h}^{-1}\right.$ ), relative speed (\%age VAM), effort (RPE), pleasure (P), desire to continue (DC), desire to stop (DS), personal resources to realized the run (R) and the ratio between the desire to continue and the effort for the group CONTROL and group RPE during the first and the second races (maximal performance was asked for the 2 groups during 2 races. During the test 1 the 2 groups totally freely control the speed. During the test 2, the group RPE has to start at least at the RPE which corresponds to the average value of the test 1 whereas the group CONTROL totally control the speed all around the run.

\begin{tabular}{|c|c|c|c|c|}
\hline & First race group CONTROL & Second race group CONTROL & First race group RPE & Second race group RPE \\
\hline Distance (m) & $737.00 \pm 122.88$ & $745.53 \pm 144.61 \mathrm{~ns}$ & $702.11 \pm 127.81$ & $\begin{array}{c}796.83 \pm 135.22^{*} \\
(+13.5 \%)\end{array}$ \\
\hline Speed (km.h $\left.\mathbf{H}^{-1}\right)$ & $14.74 \pm 2.45$ & $14.91 \pm 0.80 \mathrm{~ns}$ & $14.04 \pm 2.70$ & $\begin{array}{c}15.93 \pm 106.1^{*} \\
(+13.5 \%)\end{array}$ \\
\hline Speed (\%age VAM) & $118.80 \pm 13.28$ & $120.36 \pm 18.86 \mathrm{~ns}$ & $115.83 \pm 14.01$ & $\begin{array}{c}131.53 \pm 14.30^{*} \\
(+13.5 \%)\end{array}$ \\
\hline effort & $3.47 \pm 2.34$ & $2.75 \pm 8.04^{*}$ & $4.19 \pm 2.60$ & $6.04 \pm 1.97^{*}$ \\
\hline pleasure & $7.09 \pm 3.1$ & $8.04 \pm 2.61^{*}$ & $5.14 \pm 3.40$ & $5.42 \pm 3.29 \mathrm{~ns}$ \\
\hline Affective balance & $-3.62 \pm 4.25$ & $-5.29 \pm 3,56^{*}$ & $-0.94 \pm 5.15$ & $0.61 \pm 4.40^{*}$ \\
\hline desire to continue & $7.52 \pm 2.81$ & $8.62 \pm 1.93^{*}$ & $5.30 \pm$ & 5.32 \\
\hline desire to stop & $2.07 \pm 2.30$ & $1.29 \pm 1.83^{*}$ & $3.47 \pm$ & 3.14 \\
\hline Available resources & $7.48 \pm 2.67$ & $8.52 \pm 1.96^{*}$ & $5.79 \pm 3.08$ & $3.60 \pm 3.14 \mathrm{~ns}$ \\
\hline $\begin{array}{c}\text { Desire to continue / } \\
\text { effort }\end{array}$ & $4.46 \pm 3.61$ & $5.47 \pm 3.58^{*}$ & $2.18 \pm 2.48$ & $6.08 \pm 2.91^{*}$ \\
\hline
\end{tabular}

*: Significant difference with test $1(p<0.05)$. ns: not significantly different with test $1(p>0.05)$.

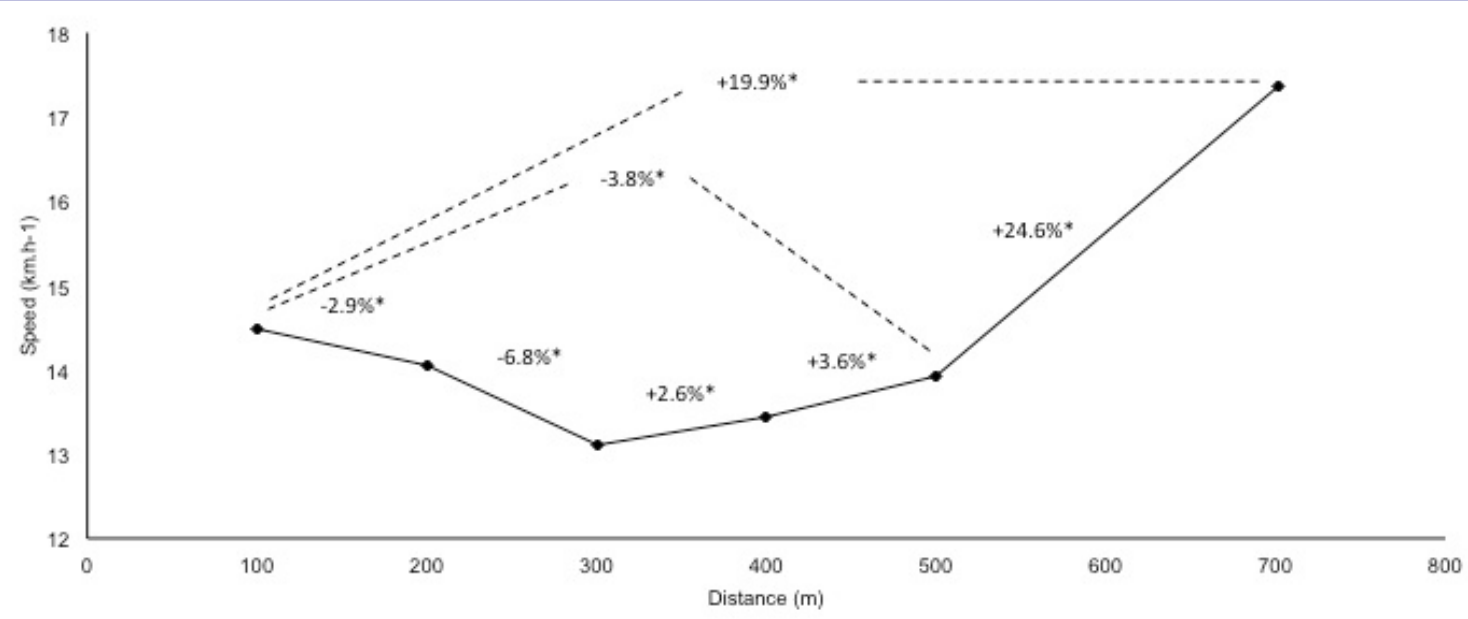

Figure 1: Evolution of self-selected speed during the first $3 \mathrm{~min}$ run of the group RPE when speed is totally self-paced:

*: Significant evolution ( $p<0.05)$; ns: not significantly evolution $(p>0.05)$.

Test 2 group RPE: Effort, affective balance and desire to stop significantly increased between the first and the last repetition $(5.02 \pm 1.42$ vs $7.87 \pm 2.19, p<0.05 ;-1.57 \pm 2.67$ vs $4.59 \pm 2.82, p$ $<0.05 ; 2.14 \pm 2.49$ vs $6.15 \pm 3.4, p<0.05$, respectively), whereas pleasure, desire to continue and available resources significantly decreased during running $(6.59 \pm 3.19$ vs $3.28 \pm 2.96, p<0.05$; $6.87 \pm 3.11$ vs $3.19 \pm 3.00, p<0.05 ; 8.27 \pm 2.06$ vs $3.45 \pm 2.96, p<$ 0.05 , respectively).

Test 1 group CONTROL: Effort, affective balance and desire to stop significantly increased between the first and the last repetition $(1.86 \pm 1.57$ vs $5.29 \pm 2.77, p<0.05 ;-6.1 \pm 2.57$ vs -0.5 $\pm 2.5, p<0.05 ; 0.93 \pm 1.54$ vs $3.71 \pm 2.93, p<0.05$, respectively), whereas pleasure, desire to continue and available resources significantly decreased during running $(7.96 \pm 3.00$ vs $5.79 \pm$ $3.30, p<0.05 ; 8.59 \pm 2.37$ vs $5.95 \pm 3.23, p<0.05 ; 8.79 \pm 1.99$ vs $5.82 \pm 3.16, p<0.05$, respectively).

Test 2 group CONTROL: Effort, affective balance and desire to stop significantly increased between the first and the last repetition $(1.93 \pm 1.62$ vs $4.02 \pm 2.46, p<0.05 ;-6.57 \pm 2.20$ vs $-2.98 \pm 2.78, p<0.05 ; 0.52 \pm 1.08$ vs $2.53 \pm 2.71, p<0.05$, respectively), whereas pleasure, desire to continue and available resources significantly decreased during running $(8.50 \pm 2.63 \mathrm{vs}$ $7.00 \pm 3.04, p<0.05 ; 9.29 \pm 1.40$ vs $7.60 \pm 2.66, p<0.05 ; 9.53 \pm$ 1.29 vs $7.38 \pm 2.68, p<0.05$, respectively). 


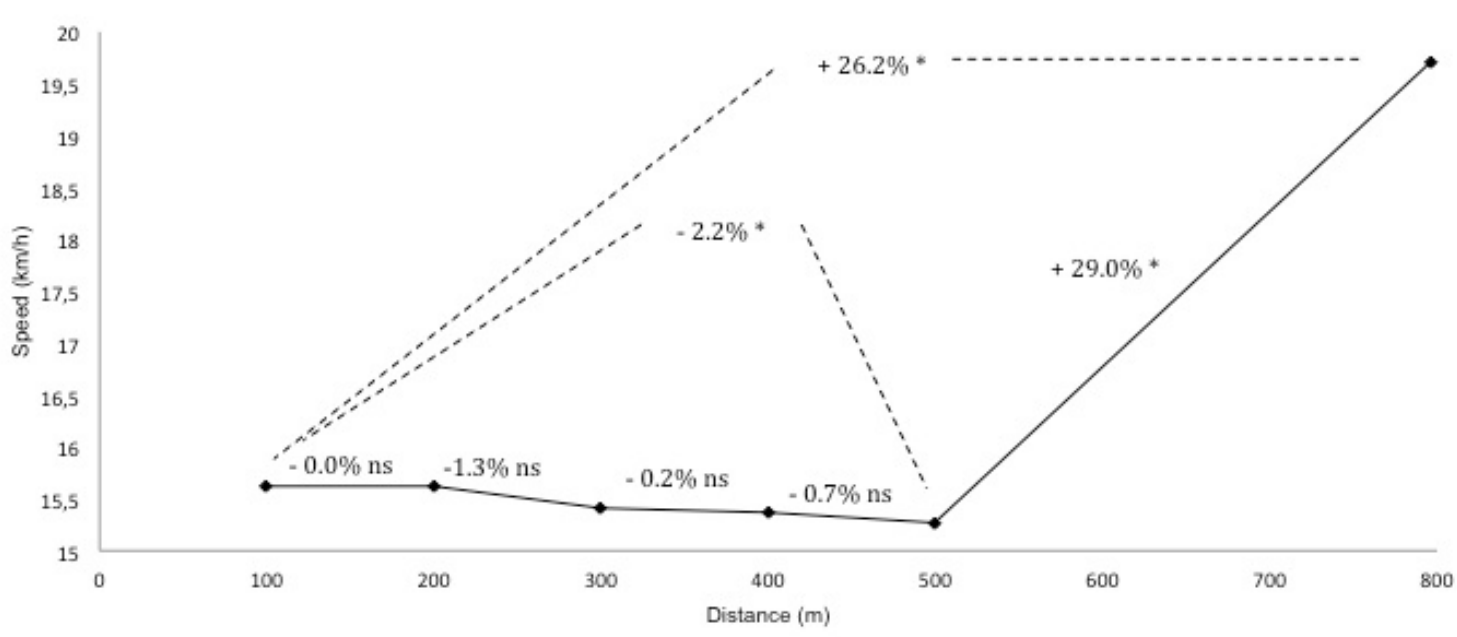

Figure 2: Evolution of self-selected speed during the second 3min run of the group RPE when speed is self-paced but the start has to be at least equal to the average RPE of the test 1 : *: Significant evolution $(p<0.05)$; ns: not significantly evolution $(p>0.05)$.

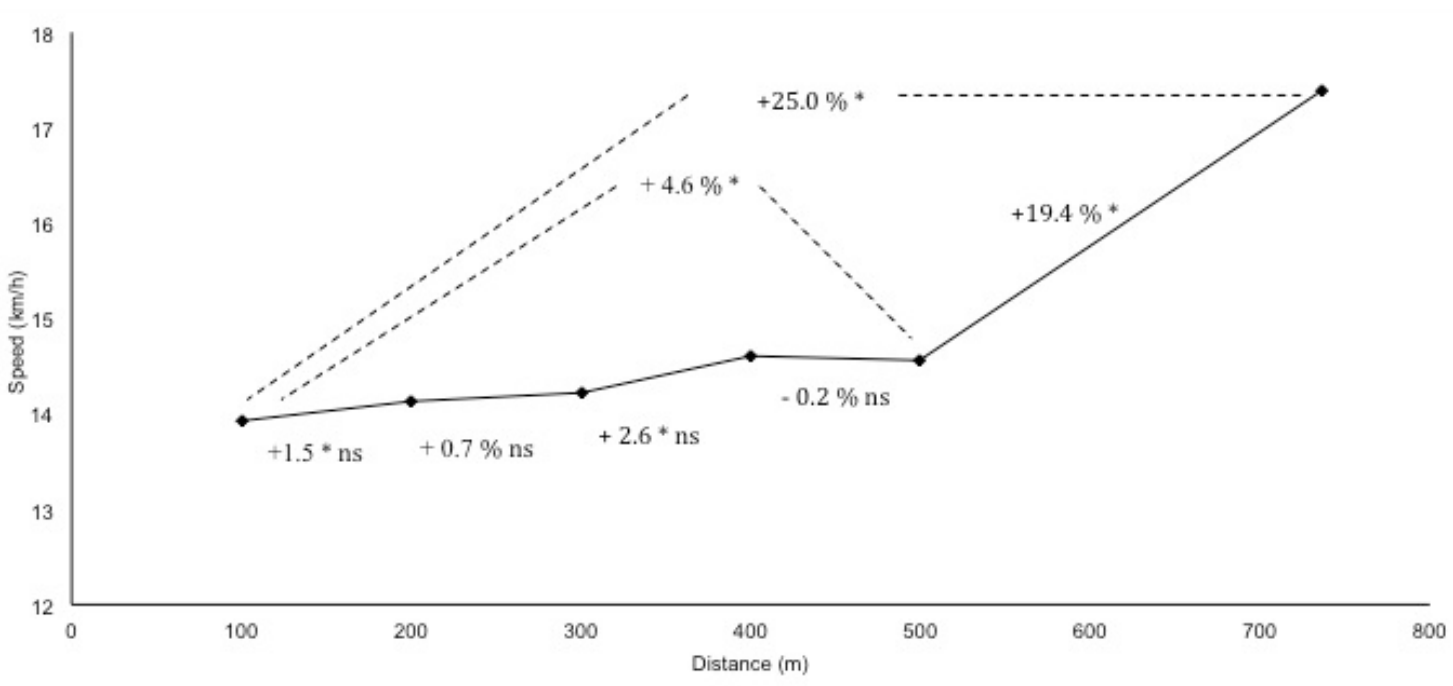

Figure 3: Evolution of self-selected speed during the first 3min run of the group CONTROL when speed is totally self-paced:

*: Significant evolution $(p<0.05)$; ns: not significantly evolution $(p>0.05)$.

Each parameter was correlated with time in each group and each test of running. During the first 3-min test of the group RPE, the duration of exercise was significantly correlated with effort, pleasure, affective balance, desire to continue, desire to stop and available resources $(0.70,-0.36,0.58,-0.43,0.44$ and -0.52 ; respectively; $p<0.05$ ).

During the second 3-min test of the group RPE, the time was significantly correlated with effort, pleasure, affective balance, desire to continue, desire to stop and available resources $(0.47$, $-0.32,0.45,-0.35,0.39$ and -0.52 ; respectively, $p<0.05$ ).

During the first 3-min test of the group CONTROL, the time was significantly correlated with effort, pleasure, affective balance, desire to continue, desire to stop and available resources $(0.50,-0.29,0.43,-0.33,0.42$ and -0.39 ; respectively, $p<0.05)$.
During the second 3-min test of the group CONTROL, the time was significantly correlated with effort, pleasure, affective balance, desire to continue, desire to stop and available resources $(0.34,-0.22,0.31,-0.27,0.23,-0.26$; respectively, $p<0.05)$.

For the CONTROL group, the slope of the relation between the time and the evolution of effort, pleasure, affective balance, desire to continue and available resources were significantly different between the first and the second 3-min run $0.04 \pm 0.03$ vs $0.02 \pm$ $0.02,-0.03 \pm 0.02$ vs $-0.02 \pm 0.02,0.06 \pm 0.04$ vs $0.04 \pm 0.03,-0.03$ \pm 0.02 vs $0.02 \pm 0.03,-0.04 \pm 0.03$ vs $-0.02 \pm 0.03$; respectively, $p$ $<0.05)$ whereas results were not significantly different for desire to stop $(0.03 \pm 0.03$ vs $0.02 \pm 0.03 ; \mathrm{p}=0.117)$. For the RPE group, the slope of the relation between the time and the evolution of effort, affective balance were significantly different between the first and the second 3 -min run $(0.06 \pm 0.03$ vs $0.03 \pm 0.02,0.10 \pm$ 


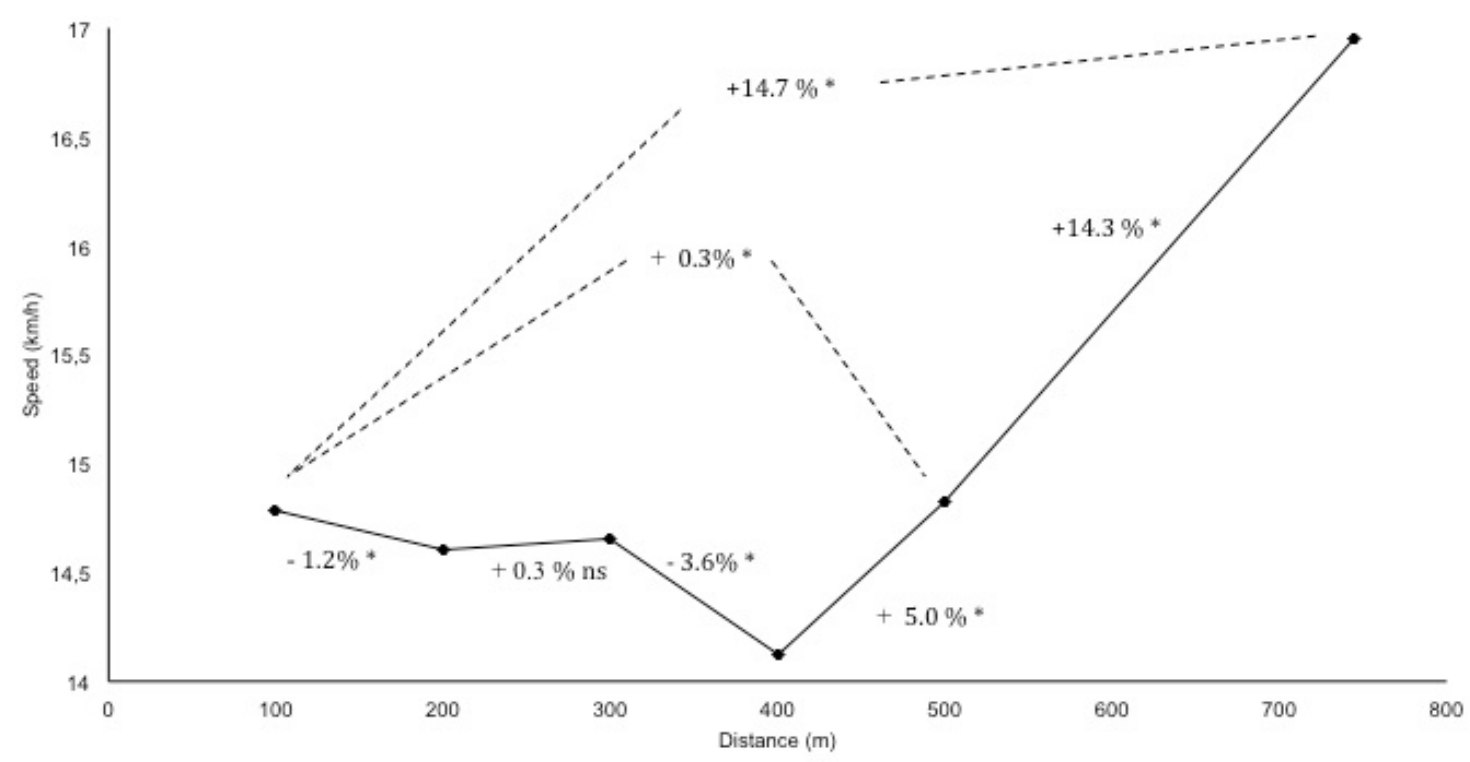

Figure 4: Evolution of self-selected speed during the second 3min run of the group CONTROL when speed is totally self-paced: *: Significant evolution $(p<0.05)$; ns: not significantly evolution $(p>0.05)$.

0.06 vs $0.07 \pm 0.05$; respectively, $p<0.05$ ) whereas results were not significantly different for pleasure, desire to continue, desire to stop and available resources $(-0.04 \pm 0.04 v s-0.04 \pm 0.03,-0.04$ \pm 0.04 vs $-0.04 \pm 0.03,0.05 \pm 0.04$ vs $0.05 \pm 0.04,-0.06 \pm 0.03$ vs $-0.05 \pm 0.03$; respectively, $p>0.05$ ).

For the CONTROL group, the Y-intercept of the relation between the time and the evolution of effort was significantly different between the first and the second 3-min run $(1.04 \pm 1.80$ vs $1.37 \pm 1.76 ; p<0.05$ ) whereas results were not significantly different for pleasure, affective balance, desire to continue, desire to stop and available resources $(8.56 \pm 3.11$ vs $8.86 \pm 2.72,-7.52 \pm$ 3.89 vs $-7.48 \pm 3.52,9.33 \pm 2.42$ vs $9.69 \pm 1.51,0.28 \pm 1.84$ vs 0.05 $\pm 1.30,9.57 \pm 2.15$ vs $9.80 \pm 1.41$; respectively, $p>0.05$ ). For the RPE group, the Y-intercept of the relation between the time and the evolution of effort and affective balance, were significantly different between the first and the second 3-min run $(0.70 \pm 1.79$ vs $4.35 \pm 1.72,-6.17 \pm 5.36 \pm-2.94 \pm 4.39$; respectively, $p<0.05$ ) whereas results were not significantly different for pleasure, desire to continue, desire to stop and available resources (6.94 \pm 4.41 vs $7.28 \pm 3.66,7.54 \pm 4.07$ vs $7.78 \pm 3.52,0.98 \pm 3.19$ vs $1.30 \pm$ 2.98, $8.86 \pm 3.52$ vs $9.19 \pm 2.52$; respectively, $p>0.05$ ).

\section{Relations between speed and emotional parameters}

The evolution of speed was not correlated with the evolution of emotional for any run and any group $(p>0.05)$.

\section{Discussion}

\section{Incremental test}

The results of the incremental test show that all the emotional parameters were correlated with the intensity whereas participants were accustomed to these scales for 1 training session, highlighting that these parameters are of interest to objectify the occurrence of fatigue.

\section{Performance and motivation during the 3-min runs}

The results of the group CONTROL show that the performance of the second 3-min run was no different of the first 3-min run whereas the comparison of the emotional parameters attests that a more important emotional reserve exist during the second run. Indeed, the average levels of effort, affective balance and desire to stop were lower whereas the average levels of pleasure, desire to continue and personal resources were higher. This could be explained by the effect of training or because participants were accustomed to this event by the first 3-min run. But it is important to keep in mind that participants were asked to realise the most important performance during the 2 running sessions. Results highlight that when participants confront the fact that enough energy is mobilized to cope with obstacles in goal attainment process and the fact that enough energy has to be conserved to reduce the probability of depletion and exhaustion, the most important parameter of the strategy is energy conservation in accordance with the theory of Gendolla and Richter [14]. It could be hypothesised that a representation of individual level of performance is developed during the first 3-min run and that even if a more important emotional reserve is observed; the level of investment is not improved in order to realise a better performance.

The results of the group RPE show that when a more important effort is imposed at the beginning of the second 3-min run, a greater distance was run. At first, this result seems obvious. But again, it is important to keep in mind that participants are allowed to pace the runs, as they wanted. Thus, they could choose to reduce or less increases the level of effort after the start. The results suggest that the performance was higher during the second run in group RPE because the emotional reserve is lower than in the first run. If effort refers to the intensity aspect of motivation [14], it could be considered that the motivation 
during the second run of group RPE was higher. Indeed, the average level of effort, affective balance and desire to stop were higher during the second run when compared with the first race.

According to Locke and Latham [25] motivation was less intense if people performed tasks without clear performance standard ("do-your-best") than when they performed a difficult task, that is, a task with clear and high performance standard. But resources are mobilized only if success is possible (i.e., not too difficult) and worthwhile (i.e., justified by the importance of success) [14].

In RPE group, starting the second run at average level of effort observed during the first run, apparently did not exceed the potential motivation, which is the maximum effort a person is (potentially) willing to mobilize for accomplishing a goal-that is the amount of justified effort [14]. Potential motivation is determined by the importance of success. The more important is succeeding, the more effort is justified.

In non-experts school participants, the knowledge of their capacity to realize the run is limited and underestimated. For this reason, the emotional reserve is important during the first run. Our results suggest that the instruction of the second run seems reinforcing the belief that the goal of a more important performance can be achieved. Because performance has direct consequences for individuals' self-definition and self-esteem [14], a more important effort is consented.

Nevertheless, even in this condition, emotional reserve is still observed and more important level of effort could have been prescribed to the participants.

\section{Pacing strategies during the 3 -min runs}

While there are an infinite number of possible pacing strategies that a subject may adopt during a run, four categories have been described by Foster et al [3], an even paced strategy, a slow start with an increase through the event. A variable paced strategy, a strategy in which the intensity is increased during the event. The last is the all-out pacing strategy in which the athlete begins at the maximal possible pace and attempts to continue. It is probably the most difficult strategy, which requires experience because the slightest mistake in the intensity at the start can induce the occurrence of a premature and catastrophic fatigue. There appears to be no clear optimal pacing strategy identified by previous research, and it may be that each individual has uniquely optimal pacing strategy [16]. Nevertheless, modelling studies have already shown that all-out performance is optimal for sprint cycling whereas benefits of a relatively fast start seem to overweigh the negative consequences of the uneven velocity profile for $1500 \mathrm{~m}$ [26-28]. For longer distances (4000m), a relatively even-paced pattern appears optimal [26].

In our study, the strategy, which consists in a slow start with an increase of speed at the end, could be observed, for each test of each group. This is principally explained by the increase of speed between $500 \mathrm{~m}$ and the last $100 \mathrm{~m}$ of the runs. It is more interesting to observe the evolution of speed between the start and the $500 \mathrm{~m}$.
The common factor that is observed when the exercise is totally self-paced (first and second runs of group CONTROL and first run of group RPE) is that fluctuations of running intensities exist between the start and the $500 \mathrm{~m}$ of exercise in untrained school participants. When a more important effort is imposed at the beginning (second run of RPE group), the intensity did not significantly evolve during this interval. It is of great interest because this strategy is known to be efficient in long duration sport events [16]. Moreover, it is probably the first strategy to master because all-out is more difficult. In addition to improved performance, the set used in the second run of RPE group improved the pacing strategy. This is of great interest in nonexperts school participants.

But the fact that the better strategy during middle duration, as 3-min runs, consists to a rapid start with a slight diminution of the speed until the finish line, suggests that a more important effort mobilisation than those corresponding to the average value of the test 1 would be more efficient on performance. The fact that an increase of the running speed was observed between $500 \mathrm{~m}$ and the end corroborate this hypothesis.

\section{Emotional strategy during the 3-min runs}

The most important finding of this study is that the pattern of emotional responses seems to be the same in all conditions. It corresponds to a quasi-maximal level of emotional reserve at the beginning and a low level at the end of exercise. It could be hypothesized that during exercise, participants compare perceived exertion with the maximum effort they are willing to mobilize for accomplishing their goal [9], i.e. more generally the level of emotional responses and the consented level in relation to the remaining time as suggested previously [8].

Moreover, the results highlight that there is a linear increase in emotional parameters between the start and the end in all conditions. This seems to be the most important foundation of the pattern of emotional regulation when participants were asked to realize the most important performance. Indeed, our results show that emotional pattern corresponded to a linear evolution in emotional parameters during the 2 runs for the 2 groups in the direction of a decrease of emotional reserve between the start and the end of the run whereas the participants were free to pace their runs, as they wanted.

It could be suggested that it is because emotional reserve is decrease throughout the runs that the speed is increased between the start and the end of the tests. Nevertheless, results show that speed was not correlated with emotional parameters for any run and any group. Indeed, we have previously suggested that emotional arousal likely takes longer to develop than do the processes involved in physiological control [8]. We proposed that a non-conscious physiological process is the most effective when rapid adjustments in power output are needed during short events. We supposed that a conscious process reinforces subconscious controls only during more prolonged exercise. The period of 3-min in these conditions of intermittent exercise appears to be insufficient for emotional regulation impact pacing strategy with significant correlation in school participants. 
The results of our previous study [15] show that the pattern of emotional regulation seems to be just translated in relation with the subjective difficulty of the task as proposed by the motivational intensity theory [14]. Indeed, Y-intercepts of the evolution of most of emotional parameters were higher when 10 repeated sprints of $60 \mathrm{~m}$ were performed on uphill surface when compared with level running. On the contrary, Y-intercepts were lower during downhill sprints. The slopes of the evolution of most of emotional parameters were not influenced by the nature of the running surface. We propose that Y-intercepts could be envisaged as a marker of the subjective difficulty of the exercise.

The results of the present research allowed us to propose the following explanatory hypothesis:

For the group CONTROL, Y-intercepts of all emotional parameters (except effort) were not significantly different between the 2 tests because the 2 runs corresponded to the same duration without any other change in the nature of the task. The slopes of the evolution of all emotional parameters (except for the desire to stop which was not significantly different) were in the way of a smaller decrease of emotional reserve during the second run. It could be explained by the theory of Gendolla and Richter [14] in which the most important parameter of the strategy is energy conservation. Hence, participants were aware to be able to attain the goal during the first run and energy conservation was the most important factor in the dilemma between the fact that enough energy is mobilized to cope with obstacles in goal attainment process and the fact that enough energy has to be conserved to reduce the probability of depletion and exhaustion [14]. Indeed, the body does not mobilize more resources than necessary [14].

For the group RPE, Y-intercepts of all emotional parameters (except effort) were not significantly different between the 2 runs for the same reason. The slopes of all emotional parameters (except RPE) were not significantly between the 2 runs. It could be hypothesized that it is because they were confronted by the same dilemma: enough energy has to be mobilized to cope with obstacles in goal attainment process but enough energy has to be conserved to reduce the probability of depletion and exhaustion. Obstacles in goal attainment process of the second run are new because the instruction was different (faster start), imposing that level of motivation was not lower than during the first test.

\section{Estimation of Motivation}

The aim of this study was not to understand the process of giving participants a reason for running so fast but rather how measuring the level of motivation which is necessary to run so fast.

According to Gendolla and Richer [14], effort refers to the intensity of motivation. But in this case, our results should be interpreted in the sense of an increase of motivation during the test, despite the occurrence of fatigue. It would also mean that participants would be more motivated when a more fast start was asked. It seems in contradiction with logic and field observations.

Rather, we proposed using other parameters to estimate the level of motivation.
We define the motivation to pursue a tired exercise as the level of acceptance of effort. The more the motivation is, the more the level of consented effort is. On the contrary, when the motivation is down, subject would not support a high level of effort. Thus, we propose that the ratio between the desire to continue the exercise and the level of effort could be used in order to estimate the motivation to pursue a tired exercise. Logically, this parameter would decrease during exercise with the occurrence of fatigue.

In accordance, our results show that this ratio decreased with time during the 3-min tests in all conditions. Moreover, this ratio was lower when a more important effort was asked at the beginning of the test (test 2 vs test 1 for RPE group) whereas it was higher when the self paced run was repeated for the second time, associated with a higher pleasure to run (test 2 vs test 1 for the CONTROL group).

\section{Conclusion}

Whereas the role of motivation is of great importance in sport performance, little is known about how to measure it during exercise. We propose that the ratio between the desire to continue the exercise and the level of effort could be used in order to estimate the motivation to pursue a tired exercise.

Whereas training programs are often designed in order to develop physiological resources, this study shows that the performance has been immediately improved by imposing a more important effort mobilization at the start without the occurrence of a catastrophic failure. Performance has been improved because a more important part of the emotional reserve has been used. Whereas many training sessions are needed in order to develop performance through an increase of physical condition, only one session was needed to improve performance with this method.

\section{References}

1. Abbiss CR, Laursen PB. Models to explain fatigue during prolonged endurance cycling. Sports Med. 2005; 35(10):865-98.

2. St Clair Gibson A, Noakes TD. Evidence for complex system integration and dynamic neural regulation of skeletal muscle recruitment during exercise in humans. Br J Sports Med. 2004; 38(6):797-806.

3. Foster C, Schrager M, Snyder AC, Thompson NN. Pacing strategy and athletic performance. Sports Med. 1994; 17(2):77-85.

4. Hulleman M, De Koning JJ, Hettinga FJ, Foster C. The effect of extrinsic motivation on cycle time trial performance. Med Sci Sports Exerc. 2007; 39(4):709-15.

5. Hettinga FJ, De Koning JJ, Broersen FT, Van Geffen P, Foster C. Pacing strategy and the occurrence of fatigue in 4000-m cycling time trials. Med Sci Sports Exerc. 2006; 38(8):1484-91.

6. Foster C, deKoning JJ, Hettinga F, Lampen J, Dodge C, Bobbert M, et al. Effect of competitive distance on energy expenditure during simulated competition. Int J Sports Med. 2004; 25(3):198-204.

7. Garcin M, Vandewalle $\mathrm{H}$, Monod H. A new rating scale of perceived exertion based on subjective estimation of exhaustion time: a preliminary study. Int J Sports Med. 1999; 20(1):40-3.

8. Baron B, Moullan F, Deruelle F, Noakes TD. The role of emotions on pacing strategies and performance in middle and long duration 
sport events. Br J Sports Med. 2011; 45(6):511-7. doi: 10.1136/ bjsm.2009.059964.

9. Ekkekakis P, Hall EE, Petruzzello SJ. Variation and homogeneity in affective responses to physical activity of varying intensities: an alternative perspective on dose-response based on evolutionary considerations. J Sports Sci. 2005; 23(5):477-500.

10. Seo MG, Barrett LF, Bartunek JM. The Role of Affective Experience in Work Motivation. Acad Manage Rev. 2004; 29(3):423-39.

11. Damasio AR. Descartes'error: Emotion, Reason and the Human Brain. New York: G. P. Putnam's Sons; 1994.

12. Damasio AR. The Feeling of what Happens: body and emotion in the making of consciousness. New York: Harcourt Incorporated; 1999.

13. Lander PJ, Butterly RJ, Edwards AM. Self-paced exercise is less physically challenging than enforced constant pace exercise of the same intensity: influence of complex central metabolic control. Br J Sports Med. 2009; 43(10):789-95. doi: 10.1136/bjsm.2008.056085.

14. Gendolla GHE, Richter M. Effort mobilization when the self is involved some lessons from the cardiovascular system. Review of General Psychology. 2010; 14(3):212-26

15. Baron B, Guilloux B, Begue M, Uriac S. Emotional responses during repeated sprint intervals performed on level, downhil and uphill surfaces. J Sports Sci. 2015; 33(5):476-86. doi: $10.1080 / 02640414.2014 .947523$

16. St Clair Gibson A, Lambert EV, Rauch LH, Tucker R, Baden DA, Foster $\mathrm{C}$, et al. The role of information processing between the brain and peripheral physiological systems in pacing and perception of effort. Sports Med. 2006; 36(8):705-22

17. Buckley JP, Sim J, Eston RG, Hession R, Fox R. Reliability and validity of measures taken during the Chester step test to predict aerobic power and to prescribe aerobic exercise. Br J Sports Med. 2004; 38(2):197205

18. Eston RG, Williams JG. Reliability of ratings of perceived effort regulation of exercise intensity. Br J Sports Med. 1988; 22(4):153-5.

19. Herbert BM, Pollatos O, Schandry R. Interoceptive sensitivity and emotion processing: an EEG study. Int J Psychophysiol. 2007; 65(3):214-27

20. Herbert BM, Ulbrich P, Schandry R. Interoceptive sensitivity and physical effort: implications for the self-control of physical load in everyday life. Psychophysiology. 2007; 44(2):194-202.

21. Foster C, Hendrickson K, Peyer K, Reiner B, De Koning JJ, Lucia A, et al. Pattern of developing the performance template. Br J Sports Med. 2009; 43(10):765-9. doi:10.1136/bjsm.2008.054841.

22. St Clair Gibson A, Foster C. The role of self-talk in the awareness of physiological state and physical performance. Sports Med. 2007; 37(12):1029-44.

23. St Clair Gibson A, Lambert ML, Noakes TD. Neural control of force output during maximal and submaximal exercise. Sports Med. 2001; 31(9):637-50

24. Borg G, Ljunggren G, Ceci R. The increase of perceived exertion, aches and pain in the legs, heart rate and blood lactate during exercise on a bicycle ergometer. Eur J Appl Physiol Occup Physiol. 1985; 54(4):3439.

25. Locke EA, Latham GP. A theory of goal setting and task performance. In: Cliffs E, editor. New Jersy: Prentice Hall; 1990.

26. de Koning JJ, Bobbert MF, Foster C. Determination of optimal pacing strategy in track cycling with an energy flow model. J Sci Med Sport. 1999; 2(3):266-77. 